\title{
A Noble Routing Protocol for Vehicular ad hoc Networks (VANETs) with Less Routing Overheads
}

\author{
Samanpreet Singh and Gagangeet Singh Aujla \\ Chandigarh Engineering College, Landran, Mohali \\ (Punjab Technical University, Jalandhar) \\ Chandigarh Engineering College, Landran, Mohali \\ (Punjab Technical University, Jalandhar) \\ gagi_aujla82@yahoo.com,samanpreetsingh1989@gmail.com
}

\begin{abstract}
Past few decades have witnessed large increase in number of vehicles on roads due to which the traffic safety needs have also become complex and hence necessary to be taken care of. Vehicular ad hoc networks (VANETs) are becoming an asset for achieving all these goals. VANETs provide applications like driver assistance, passenger comfort and vehicle safety. Apart from its general characteristics due to the dynamic nature of road environments and traffic patterns, communication linkage among vehicles in VANETs suffers breakage problem hence requires a very reliable routing protocol. To manage the communication efficiently many routing protocols are already available for MANETs which can be further used for routing and communication among vehicles in VANETs, but characteristics of these two networks (VANET and MANET) are very different, so there is requirement to enhance these MANET routing protocols so that those can be used in VANETs. When we use some existing routing protocols in VANET then number of link breakage are surprisingly high because mobility is higher in VANETs as compared to MANETs. So this paper presents advanced version of an existing MANET routing protocol for better performance in VANET environment. The new routing protocol has removed problem of large number of routing error messages which was existing in AODV routing protocol. Protocol proposed in this paper has better results as compared to AODV and paper has also shown results of simulation and also elaborated the findings.
\end{abstract}

Keywords: VANET, MANET, AODV, AODV-FN, Simulation, Routing protocol

\section{Introduction}

With the advent of Intelligent Transportation System (ITS) [1], automobile companies have started deploying more Electronic Control Units (ECUs) in vehicles for engine management, air bag controlling, automatic brake systems and air condition control. Along with these enhancements the increase in vehicles on roads and streets has also made it compulsory to implement safety applications for vehicles. Vehicular Ad Hoc Networks (VANETs) [2] are gaining scope because they are providing numerous applications like traffic safety, driver assistance, entertainment, internet access, automatic toll payment and many more for roadside environments.

Vehicular Ad Hoc Networks (VANETs) have similarities to Mobile Ad Hoc Networks (MANETs) like random topology and short communication range. These two characteristics depict that messages could not be directly delivered to destinations rather a message shall be routed by intermediate nodes to given destination. So routing protocol is very important in 
both VANET and MANET environments and almost having same requirements with some changes. But the second side of coin, there are some major differences which are also available for these two networks. In Mobile Ad Hoc Networks (MANETs) environments mobile nodes can move in any direction without any geographical and spatial constraints. But in Vehicular Ad Hoc Networks (VANETs) mobile nodes have to follow some patterns because of roads, streets and buildings in their way of movement. Second difference in VANETs is that the connectivity among vehicles, size of network and speed of node are highly dynamic as compared to MANETs.

Thus by doing some little changes to MANET routing protocols, these protocols can be used for better performance in VANETs. This paper has presented new routing protocol for VANETs by modifying the existing MANET routing protocol AODV for achieving better performance in terms of routing overheads, no. of advertisements and packet delivery ratio. Second section of this paper highlights literature survey which explains the performance comparison of various MANET routing protocols. Third section of this paper give details regarding MANET routing protocols, section four elaborates new routing protocol AODV-FN (Forget Neighbors), design and implementation of new routing protocol is given in next section. Simulation results for various parameters of network performance are given in the following section and final section contains conclusion and future scope of this research.

\section{Literature Survey}

Asma et al. [3], compared DSR, AODV and DSDV. DSDV has higher routing load than AODV and DSR but has lower throughput than both of these. AODV and DSR have performed well in all comparisons but in some cases AODV outperforms DSR. But DSR was better when evaluated in terms of average end to end delay. Changing the packet size has affected the performance of AODV and DSR but had no effect on DSDV. Authors of Monarch project [4] have evaluated DSR, DSDV, AODV and TORA in terms of periodic advertisements, source routing, on demand route discovery and hop by hop routing. They have observed that DSR performs well for all parameters.

In [5], authors compared AODV and DYNMO protocols for path optimally, routing overheads, packet delivery ratio, throughput and end to end delay. In these comparisons DYNMO shows better performance than AODV because it can handle different traffic patterns and mobility conditions. Paul et al., [6], have done analysis of AODV and DSR in VANET environment in terms of node speed and node density. Higher packet delivery ratio (PDR) has been recorded in lower density and lower speed. Packet loss has been also decreased. Author compared AODV and DSR with respect to CBR and TCP. Packet delivery ratio of AODV is average under high speed conditions.

\section{Routing Protocols for MANETs}

Three main classes of routing protocols which are available for MANETs: first, proactive routing protocols; second is reactive routing protocol and last one is hybrid routing protocols.

\subsection{Proactive Routing Protocols}

In this category of routing protocols a table is maintained for every reachable node in the network. These protocols update this table time to time. Advantage of this kind of routing protocol is that whenever a node wants to send data it can find path to destination node very easily. The examples of these kinds of protocols are Fisheye State Protocol (FSR) [7], 
Optimized Link State Routing (OLSR) [8] and Topology Dissemination Based on Reverse Path Forwarding (TBRPF) [9].

Optimized Link State Routing (OLSR) is routing protocol in which node routing table maintains paths to all nodes of network and at the time of change in topology it changes the tables according to change occurred. So due to this characteristic of this protocol it is not appropriate for highly dynamic VANET environments. On the other hand Fisheye State Protocol (FSR) is good for VANET environment because it does not send message on link failure. But major drawback of FSR is its processing time and storage needs for routing table. In Topology Dissemination Based on Reverse Path Forwarding (TBRPF) each node periodically updates the paths to other nodes by sharing control messages to each other. Problem in this protocol is flooding of control message whenever a change occurs.

\subsection{Reactive Routing Protocol}

In this category of routing protocols path to destination is searched only when its need arises. So these protocols are also known as "on demand" routing protocols. Search to the destination node ends only when link to that node found or the path to given node is not available from the current node.

Major difference in reactive and proactive routing protocols is that, routing overheads for reactive routing protocols are very less because they find a path to a node only when it has demanded. So a little overhead occur on control messages. This is reason why proactive routing protocol cannot be used in VANETs where topology changes very frequently. Examples of protocols based on reactive strategy are: Temporally Ordered Routing Algorithm (TORA) [10], Dynamic Source Routing (DSR) [11], protocol and Ad hoc On Demand Distance Vector (AODV) [12] routing protocol.

In Dynamic Source Routing (DSR), link to the destination is searched and established only when requested to do so. But this link maintenance method is not able to repair broken link and performance of protocol decreases when mobility is very high. In Temporally Ordered Routing Algorithm (TORA), when number of nodes increase more than thirty then performance of this protocol degrades. So this protocol is suitable only for small networks. AODV from reactive protocol category is best suitable for mobile ad hoc networks. A path from source to destination is determined only on demand and paths to all nodes are not maintained. The main benefit of using AODV routing is that it uses sequence numbers to check the freshness of the route. And this is also a loop free protocol and is suitable for different sizes of networks and high mobility conditions. It comes to market with different implementations like AODV-UU [13] and Kernel-AODV.

\subsection{Hybrid Routing Protocols}

Hybrid routing protocol category contains both proactive and reactive features. These are designed in such a manner that scalability of proactive routing protocol is improved by lowering the routing overheads. In this various nodes which are close to each other to make backbone for network so that routing overheads can be minimized. Zone Routing Protocol (ZRP) [14] is type of hybrid routing protocol. In this whole network is divided into zones of different sizes. It uses reactive protocol strategy in inter-zone environment and uses proactive technique for intra-zone route discovery. It suffers from the latency problem while finding new routes.

In our paper reactive routing protocol is used because it does not store routing table for whole network hence uses less memory which is very suitable for VANETs. Because 
embedded systems for vehicles have very less memory. That's why protocol which uses more memory for routing table storage is not so effective in VANET environments.

\subsection{Ad hoc On Demand Distance Vector (AODV) Routing Protocol}

Ad hoc On Demand Distance Vector (AODV) [15] routing protocol is best routing protocol for VANET environment because it uses less memory for routing table storage. Second reason for choosing AODV is that it is available in Network Simulator 2 (NS2) [16]. AODV uses four major messages for their working:

1) RREQ (route request message)

2) RREP (route reply messages)

3) HELLO (notifications)

4) RERR (routing error messages)

RREQ is used for route discovery to a given destination. RREP is a reply from destination node or from intermediate node which has a valid route to the destination. HELLO messages are sent on regular intervals to find active neighbors. And RERR messages are sent to source node when a link is broken on which it had sent some packet.

There is no problem with first three types of messages but fourth type of messages is very problematic in AODV routing protocol. Because it is generated when there is a link failure in the network. VANETs are very dynamic in nature so link loss is very high due to high speed and mobility of vehicles. So these messages in AODV are major source of congestion. This paper has explained this problem in next section and solved it by new routing protocol AODV-FN.

\section{Problem Explanation and Proposed Work}

\subsection{How RERR arising problems in AODV}

AODV sometimes uses old links to its neighbors for sending the packets. Most of the times neighbors change their location very frequently due to high speed so their old paths too those neighbors become invalid but AODV is unaware of it. In AODV nodes use high value for expiry for their neighbors so the paths to these neighbors are marked as valid in node's local table until the RERR message arrive but in reality those paths are invalid.

So whenever a source node sends packets to its neighbors for forwarding them to destination, in response to this RERR messages are generated about unavailability of that path, because neighbors are not present at their old positions and they have moved to some other location. Now on reception of RERR source node mark those paths invalid in its routing table and sends HELLO message for new neighbors and on receiving REPLY HELLO it again finds path to destination by sending fresh RREQ message to new neighbors. Now suppose time taken in arrival of RERR from broken link is $\mathbf{t}_{\text {error. }}$ Time taken to find neighbor by HELLO and time taken to search new route to destination are $\mathbf{t}_{\text {hello }}$ and $\mathbf{t}_{\text {newroute }}$ respectively, then total time to find route to a node is $\mathbf{t}_{\text {total }}$ :

$$
\mathbf{t}_{\text {total }}=\left(\mathbf{t}_{\text {hello }}+\mathbf{t}_{\text {newroute }}\right)
$$

But total time taken for finding new route after an error is $\mathbf{t}_{\mathbf{a f t e r e r r o r}}$ :

$$
\mathbf{t}_{\text {aftererror }}=\mathbf{t}_{\text {error }}+\left(\mathbf{t}_{\text {hello }}+\mathbf{t}_{\text {newroute }}\right)
$$


so extra time taken due to error in link loss is $\mathbf{t}_{\text {error. }}$ This time is for one request only but there are many RERR messages in whole network. Suppose total number of errors in network is $E_{n}$ then total extra time for network is $\mathbf{t}_{\text {extra }}$ :

$$
\mathbf{t}_{\text {extra }}=\left(\mathbf{E}_{\mathbf{n}} * \mathbf{t}_{\text {error }}\right)
$$

This time is very high which reduces packet delivery ratio (PDR) of AODV and also increases the routing overheads due to large number of RERR messages. The main reason behind all these RERR messages is high neighbor expiry time due to which nodes uses old links to their neighbors which are not at their previous known locations due to their mobility.

\subsection{Proposed Work}

The problem in AODV was, it used to maintain the neighbors which were intermediate nodes in recent packet sending event of current node. It does not invalidate its neighbors very quickly and trust the last few paths used in packet delivery process but VANET environments are highly dynamic so all near nodes are changing their locations very fast. To solve this problem of the AODV this paper had proposed a new protocol AODV-FN (AODV-Forget Neighbors) which reduces the neighbor's expiry time for a node's neighbors. Before going to that we should understand some key terms used in AODV.

\subsubsection{Neighbor expiry}

It is time after which a source node concludes that its neighbor has expired and it changes the validation for old paths to that node. But it does not mean that if that node is present in our neighborhood we will not consider it our neighbor.

\subsubsection{Allowed Hello loss}

Allowed hello loss means number of HELLO messages protocol is assuming to fail due to physical characteristics of network. This is generally very less in various protocols because HELLO messages have very less failure rate.

\subsubsection{Current time}

Current time is real world time of environment in which vehicle is moving. This is generally taken form $\mathrm{C}++$ scheduler function in NS 2 simulator.

\subsubsection{Hello interval}

It is time gap after which a node sends HELLO messages to its neighbors for its presence and to know their presence in the neighborhood. It should not be too small because it will reduce the bandwidth of network by sending large number of HELLO messages. It should not too large because it will reduce the performance by insufficient number of HELLO messages.

Below given line describe how we have reduced neighbor expiry time: (IV)

nb_expire $=$ current time $+(.25 *$ ALLOWED_HELLO_LOSS $*$ HELLO_INTERVAL $)$

New protocol AODV-FN will perform better than the old one because of following changes: 
-Each node will find new neighbors of it at present time and use them to discover fresh paths to destination. It will store them in its list for time given in equation (iv). This process will repeat for $\mathrm{n}$ nodes in network without leading to erroneous old paths.

- Now recent routes will not be used by the node because on expiry of neighbors it will invalidate all the old routes to those neighbors. Maintenance of table is for less time as compared to AODV.

- Each time precursor list will not be followed because it can have broken paths, those can lead to error messages. Less RERR messages will be generated because each time protocol will find new trustworthy paths to destinations.

- Total number of $E_{n}$ (no. of errors across network) as given in equation (iii) will be reduced. Due to this total extra time $\mathbf{t}_{\text {extra }}$ will be almost nil.

All these changes will help new protocol to enhance performance in terms of Packet Delivery Ratio (PDR), Advertisements and Routing load. All these parameters are improved in new routing protocol AODV-FN as compared to existing AODV routing protocol. The results of simulation in different traffic conditions have shown in section sixth.

\section{Design and Implementation}

For implementation of above said routing protocol we have used mainly two simulators: SUMO (Simulation of Urban Mobility)

Network Simulator 2 (NS2).

\subsection{SUMO (Simulation of Urban Mobility)}

One of the foremost requirements while simulating VANETs is that we should keep the mobility model as real as possible. Real world traffic environment contains roads, junctions, traffic lights, dead ends and crossings. So these all real world aspects should be preserved in simulation. For this purpose we have chosen SUMO [17]. SUMO is a freeware simulator for simulation of real time traffic environments. It can import maps from various sources like OpenStreetMap, Tiger database and from many other sources. In our implementation we have taken map from OpenStreetMap. Many maps were shortlisted in beginning but in the end a map with good combination of straight lines and intersections has been chosen.

Then mobility trace file for this traffic environment which could be run in NS-2 has been created with the help of MOVE which is built on top of SUMO. Because SUMO can create simulation environment for real road network but could not show communication among vehicles. Simulation screenshot of SUMO is given in following Figure 1.

\subsection{NS2 (Network Simulator 2)}

NS2 is a popular open source tool which based on 802.11 MAC layer of wireless communication networks. It is an object oriented tool which runs on occurrence of events in the network. We have used ns-2.35 version of NS-2 in which AODV is built in. NS2 can run .tcl files generated from real time traffic in SUMO and can show visual output of communication and message passing with the help of nam (network animator). 


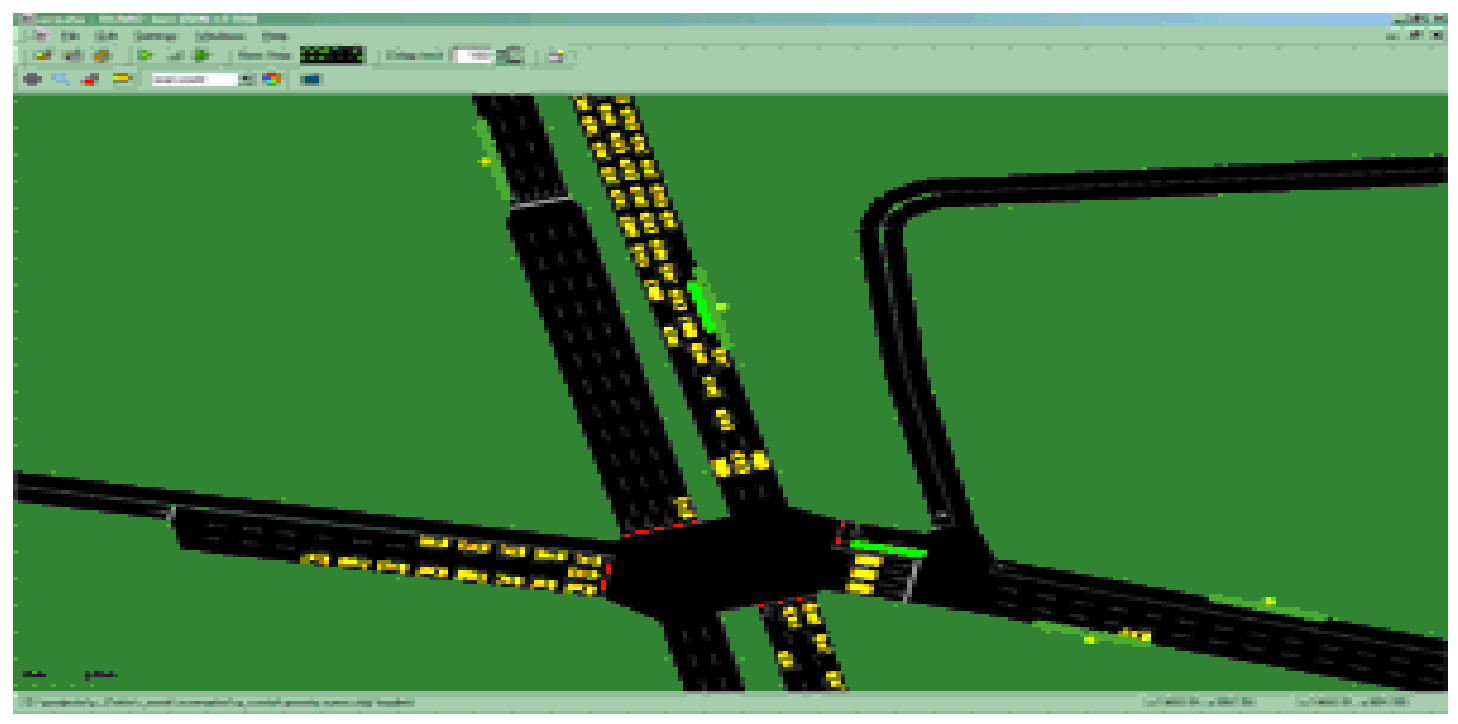

Figure 1. SUMO Simulation Environment

Various steps of simulation are shown in blew figure.

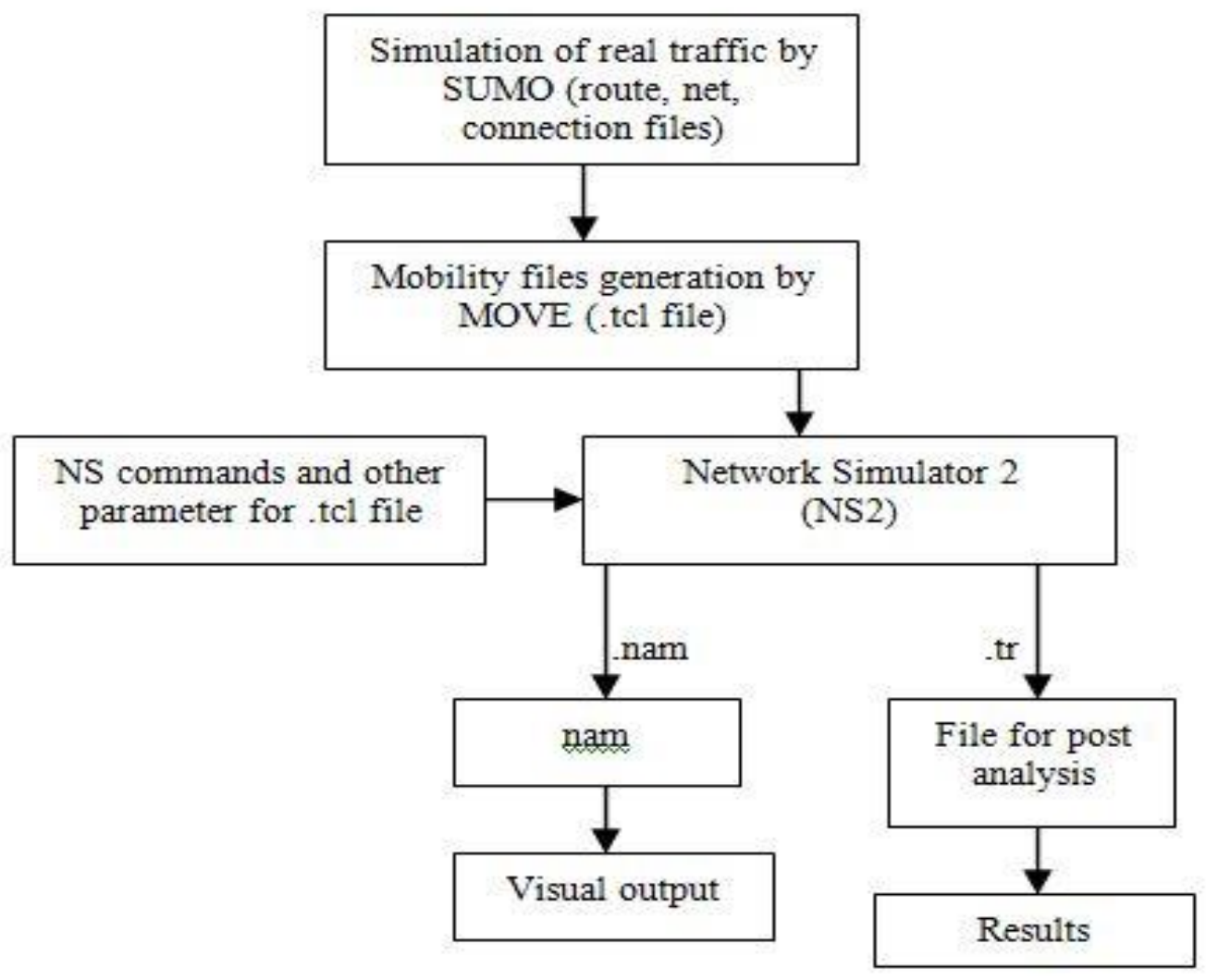

Figure 2. Implementation Steps 
Step 1: In first step SUMO is used for simulation of real traffic. Different files are generated during this simulation process like .net (network files), .rou (route files), .con (connection files) etc.

Step 2: In this step MOVE which is a part of SUMO is used to convert SUMO output files to .tcl (mobility file), because NS2 could run only these .tcl files.

Step 3: Now NS2 uses .tcl file to show communication environment using .nam file on nam console. Second file generated by NS2 is .tr (trace file) which contains various parameters of simulation like generated TCP packets, dropped packets, sending time, receiving time and many other aspects of communication. In NS2 we can apply different routing protocols on traffic (AODV, AODV-FN in our case).

Step 4: Now from output files of NS2 we can see communication by nam using nam console or can generate different results from tr file.

In this way by applying AODV and new routing protocol AODV-FN we have generated various .tr files and then used them to conclude results for routing load, advertisements generated and packet delivery ratio. These results are shown in next section. In NS-2 parameters given below in table 1 are used to compare results of AODV and new routing protocol AODV-FN.

Table 1. Simulation Parameters

\begin{tabular}{|l|l|}
\hline Parameter & Value \\
\hline Simulation time & $110 \mathrm{~s}$ \\
\hline Routing protocols & AODV, AODV-FN \\
\hline NS-2 version & Ns-2.35 \\
\hline Communication range & $550 \mathrm{~m}$ \\
\hline Packet size & 1024 \\
\hline Map topology & $1652 \mathrm{~m}$ x1652m \\
\hline Map source & Openstreetmap \\
\hline TCP sources & $\begin{array}{c}4 \text { pairs of nodes with } \\
\text { TCP } \\
\text { and TCP sink. }\end{array}$ \\
\hline
\end{tabular}

\section{Simulation Results}

Each simulation result is compared for different number of nodes 24,40 and 60. Different numbers of node are taken to check performance for different traffic conditions. Comparison has done in terms of routing load percentage, number of advertisements generated and packet delivery ratio.

Routing packets are number of routing information messages generated by a protocol to manage communication. These are used to check availability of neighbors and error reporting messages. Now advertisements are number of routing messages generated by protocol during total simulation time. On the other hand, routing load is calculated from number of advertisements divided by total number of messages (routing messages + data messages) generated during simulation time. It is clear from above definition that routing load percentage should be less for better performance of protocol 


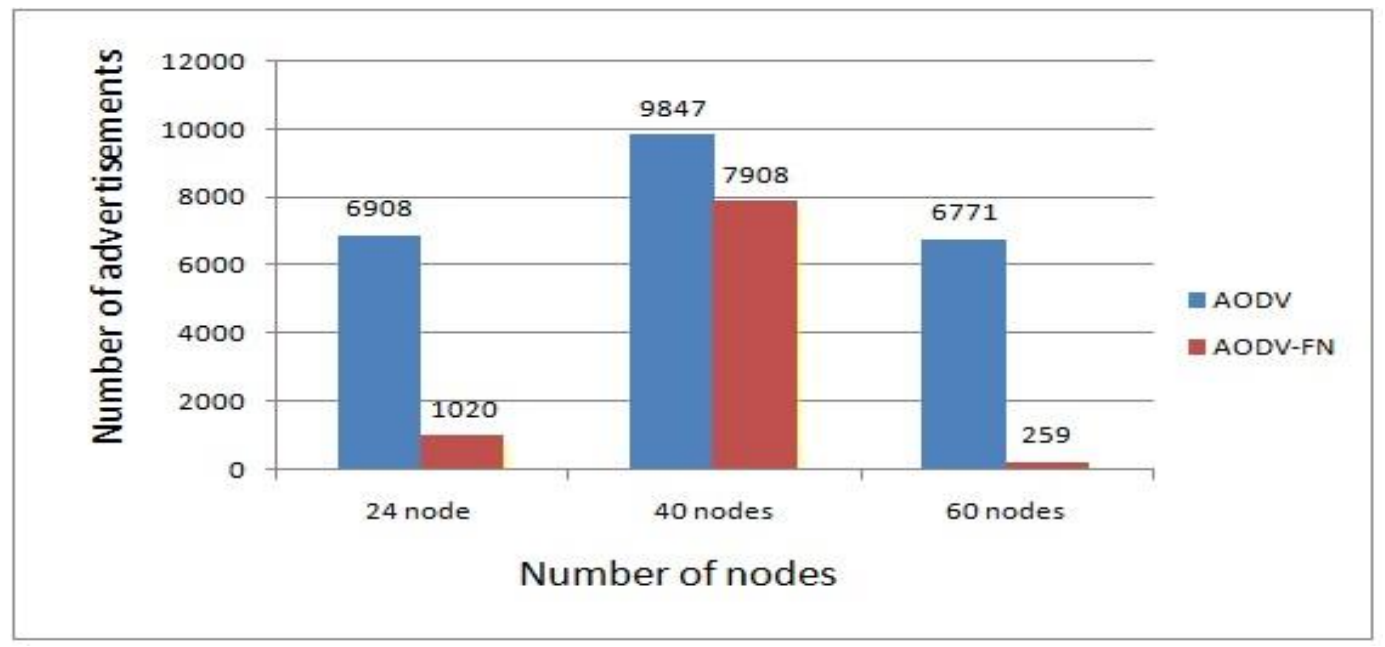

Figure 3. Number of Advertisements

In chart of Figure 3 AODV-FN has better results than AODV because it generates less number of advertisements in all the three different scenarios as compared to AODV. For 60 nodes performance of new routing protocol is very high just 259 advertisements have generated, which shows it generates very less advertisements in high traffic conditions.

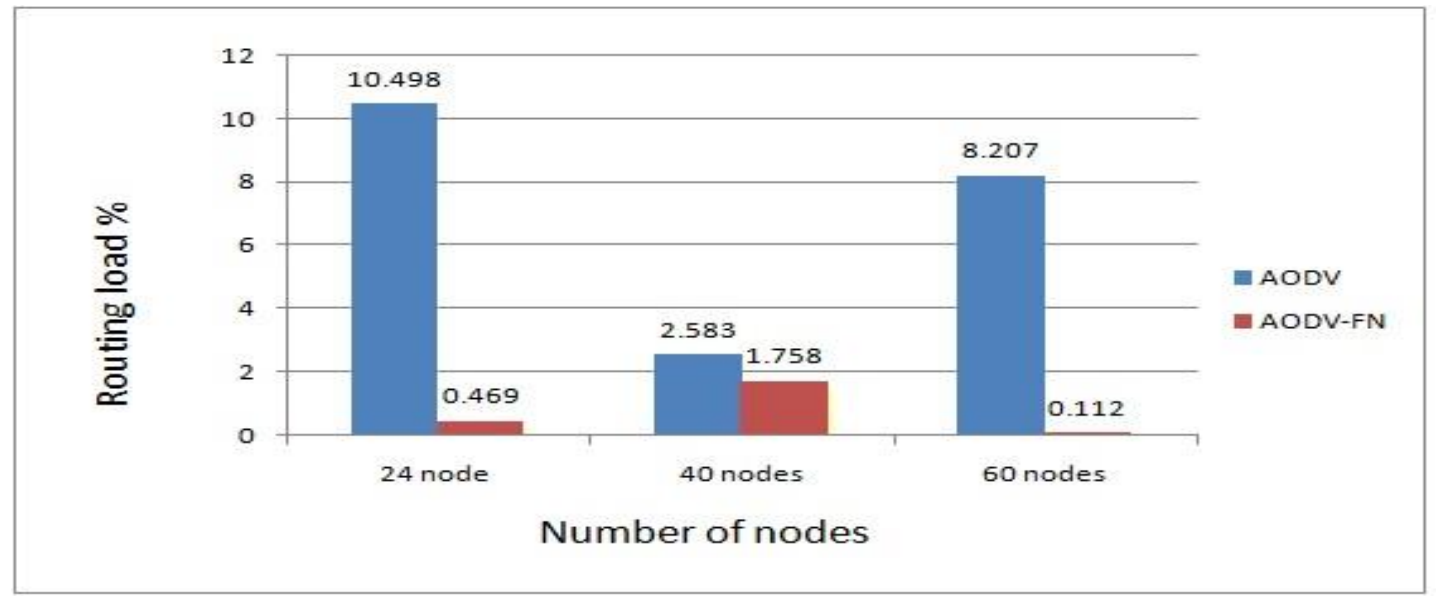

Figure 4. Routing Load \%

In Figure 4 routing load of AODV-FN is less in every case for different number of nodes. For 24 nodes and 60 nodes it is below 1\%, it means our new routing protocol will provide more bandwidth for data packets which will improve the communication among nodes without suffering from any bandwidth limitation. Due to less routing load in AODV-FN packet delivery ratio of new protocol has increased as compared to AODV. Below given Figure 5 shows packet delivery ratio for both these protocols for different number of nodes. For 24 nodes and 60 nodes AODV-FN's PDR is more by approximately $6 \%$ and $9 \%$, which is huge difference as compared to AODV. Results of advertisements, routing load and packet delivery ratio show how by reducing RERR messages in old protocol our performance has been improved. 


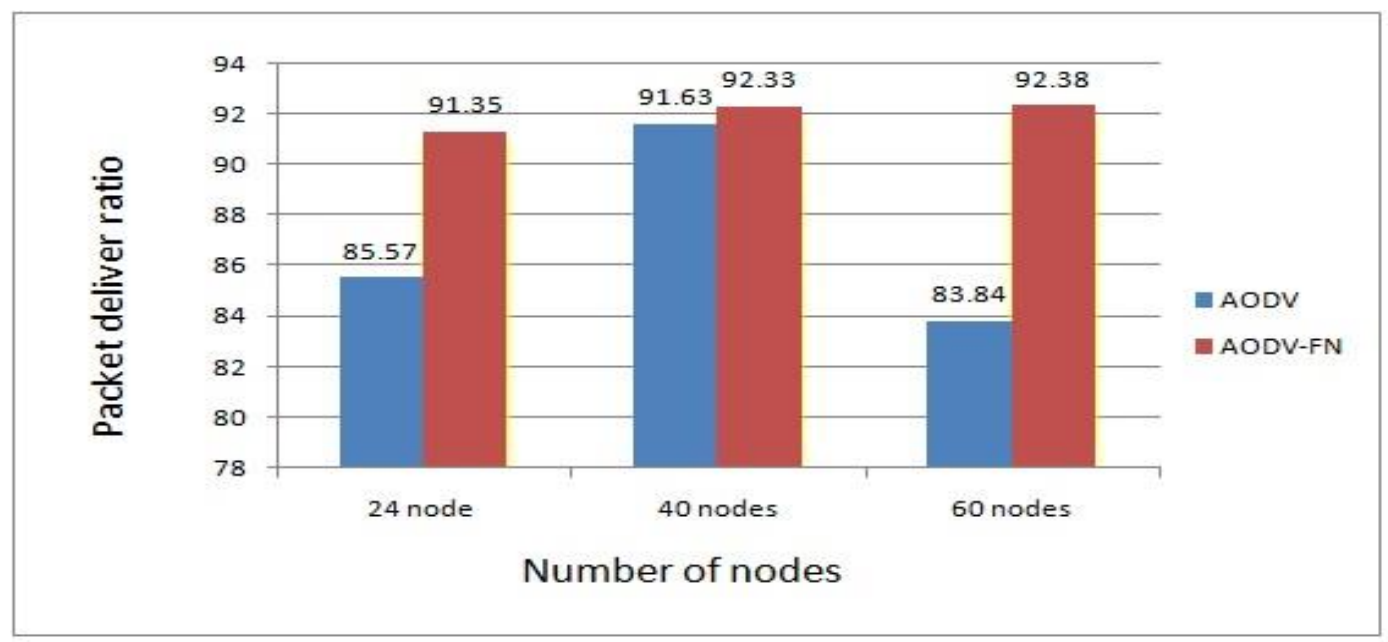

Figure 5. Packet Delivery Ratio

\section{Conclusion and Future Work}

This paper has presented an enhanced version of existing MANET routing protocol AODV by reducing neighbor expiry time, which shows dramatic improvements in performance of old routing protocol. Due to less expiry time for neighbors source nodes are using the broken paths very less, because after a short time it assumes that its neighbor has been moved to another location and it invalidates all paths to various destinations through that neighbor. Now each time originator of the message finds new trustworthy neighbors and sends packets via them without any error. In this way we have designed AODV-FN, a new routing protocol that has less routing overheads and generating fewer advertisements than AODV. Packet delivery ratio of new routing protocol is also improved.

In future we can enhance the performance of this protocol to higher peaks by adding new features according 802.11 MAC layer. Researcher can also suppress HELLO messages and can stop their blind broadcasting. These enhancements can further improve the performance of AODV.

\section{References}

[1] T. Taleb, E. Sakhaee, A. Jamalipour, K. Hashimoto, N. Kato and Y. Nemoto, “A Stable Routing Protocol to Support ITS Services in VANET Networks", IEEE transaction on vehicular technology, vol. 56, no. 6, (2007) November, pp. 3337-3347.

[2] J. Nzouonta, N. Rajgure, G. (Grace) Wang and C. Borcea, "VANET Routing on City Roads Using RealTime Vehicular Traffic Information", IEEE transaction on vehicular technology, vol. 58, no. 7, (2009) September,pp. 3609-3626.

[3] T. Asma, G. Rajneesh and T. Sunil and, "Comparative Performance Analysis of DSDV, AODV and DSR Routing Protocols in MANET using NS2", in International Conference on Advances in Computer Engineering, Bangalore, Karnataka, India, (2010).

[4] D. A. Maltz, D. B. Johnson, Y. Hu, J. Broch and J. Jetcheva, "A Performance Comparison of Multi-Hop Wireless Ad Hoc Network Routing Protocols", in Fourth Annual ACM/IEEE International Conference on Mobile Computing and Networking, Ballas, Texas, (1998).

[5] M. Quan-xing and X. Lei, "DYMO Routing Protocol Research and Simulation Based on NS2", in International Conference on Computer Application and System Modeling, Taiyuan, Shanxi,China, (2010).

[6] B. Paul, Md. Ibrahim and Md. Bikas, "Experimental Analysis of AODV \& DSR over TCP \& CBR Connections with Varying Speed and Node Density in VANET", International Journal of Computer Applications, vol. 24, no. 4, (2011) June. 
[7] G. Pei, M. Gerla and T.-W. Chen, "Fisheye State Routing in Mobile Ad Hoc Networks", in Proceedings of the 2000 ICDCS Workshops, Taipei, Taiwan, (2000).

[8] K. V. Patil and M. R. Dhage, "The Adaptive Optimized Routing Protocol for VehicularAd-hoc Networks", IJCSN International Journal of Computer Science and Network, vol. 2, no. 3, (2013) June, pp. 67-70.

[9] V. U. Chezhian, K. Karthikeyan and T. Subash, "Comparison of Two Proactive Protocols: OLSR and TBRPF using the RNS (Relay Node Set) Framework", International Journal of Computer Science \& Emerging Technologies, vol. 2, April (2011), no. 2, pp. 324-329.

[10] V. D. Park and M. S. Corson, "A Performance Comparison of the Temporally-Ordered Routing Algorithm and Ideal Link-State Routing”, in IEEE, Athens, (1998), pp. 592 - 598.

[11] R. Singh, D. K. Singh and L. Kumar, "Performance Evaluation of DSR and DSDV Routing Protocols for Wireless Ad Hoc Networks", Int. J. Advanced Networking and Applications, vol. 02, no. 04, (2011) October, pp. $732-737$.

[12] A. Odeh, E. A. Fattah and M. Alshowkan, "Performance evaluation of AODV and DSR routing protocols in MANET networks", International Journal of Distributed and Parallel Systems (IJDPS), vol. 3, no. 4, (2012) July, pp. 13-22.

[13] P. K. Maurya, G. Sharma, V. Sahu, A. Roberts and M. Srivastava, "An Overview of AODV Routing Protocol”, International Journal of Modern Engineering Research (IJMER), vol. 2, no. 3, (2012) May, pp. 728-732.

[14] V. Mallaiah, Dr. A. VinayaBabu, K. Madhukar, S. Nagaprasad, D. M. G. Verghese and A. Sreelatha, "Multicast zone routing protocol in mobile ad hoc networks", International Journal of Engineering Science and Technology, vol. 2, no. 10, (2010), pp. 5647-5656.

[15] C. Perkins, E. Belding-Royer and S. Das, "Ad hoc On-Demand Distance Vector (AODV) Routing”, Network Working Group , Experimental rfc3561 , (2003).

[16] "network simulator-2 retrieved from http://www.isi.edu/nsnam/ns/,".

[17] "SUMO (SIMULATION OF URBAN MOBILITY) retrieved from on 12 feburary 2014 ttp://sourceforge.net/apps/mediawiki/sumo/index.php?title=Main_Page,".

\section{Authors}

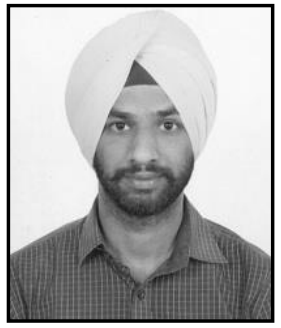

Samanpreet Singh, he is a student of Master of Technology in Computer science engineering at Chandigarh Engineering College, Landran. He has received his Bachelor of Technology degree in Information Technology from Punjab Technical University, Jalandhar.

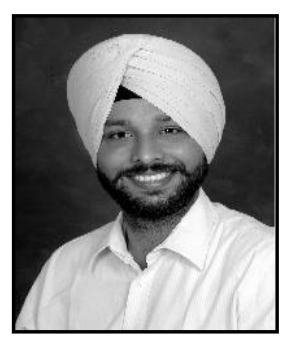

Gagangeet Singh Aujla, he is working as Assistant Professor at Chandigarh Engineering College, Landran. He has been teaching from last 8 years in the field of Computer Science \& engineering. He is active member of Computer Society of India and Indian national Science Congress. He did his $M$. Tech from Punjab technical university. He has about 12 research papers published in International Journals/Conferences. 
International Journal of Future Generation Communication and Networking Vol.7, No.5 (2014) 